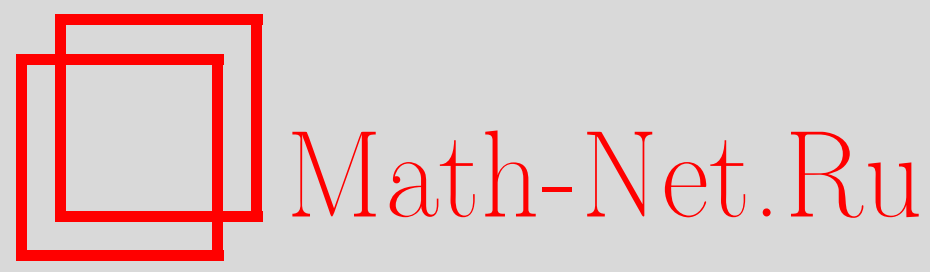

И. А. Чельцов, Поверхности Дель Пеццо с нерациональными особенностями, Матем. заметки, 1997, том 62, выпуск $3,451-467$

DOI: https://doi.org/10.4213/mzm1627

Использование Общероссийского математического портала Math-Net.Ru подразумевает, что вы прочитали и согласны с пользовательским соглашением http://www.mathnet.ru/rus/agreement

Параметры загрузки:

IP : 3.85 .5 .30

26 апреля 2023 г., 16:26:09 


\section{ПОВЕРХНОСТИ ДЕЛЬ ПЕЦЦО \\ С НЕРАЦИОНАЛЬНЫМИ ОСОБЕННОСТЯМИ}

\section{И. А. Чельцов}

В данной работе классифицируются нормальные алгебраические поверхности $X$ c $\operatorname{rk}(\operatorname{Div}(X) \otimes \mathbb{Q} / \equiv)=1$ и численно обильным каноническим классом, имеющие нерациональные особенности. В частности, доказывается, что все такие поверхности $X$ есть стягивание "исключительного" сечения на возможно особой "относительно минимальной" линейчатой поверхности $\widetilde{X}$ с нерациональной базой. Более того, $\widetilde{X}$ однозначно определяется исходной поверхностью $X$.

Библиограффия: 6 названий.

Введение. Работы $\Phi$. Сакая естественным образом переносят вопросы классификации алгебраических поверхностей в категорию нормальных алгебраических поверхностей. На таких поверхностях можно формально определить численный обратньй образ дивизора Вейля, который обладает хорошими функториальными свойствами и позволяет построить пересечения $\mathbb{Q}$-дивизоров Вейля над $\mathbb{Q}$ (см. [1]). Численные поверхности Дель Пецшо и "относительно минимальные" линейчатые поверхности играют в классификации $\Phi$. Сакая ту же роль, что и гладкие поверхности с размерностью Кодаиры $-\infty$ в классификации гладких алгебраических поверхностей.

Заметим, что в работе [2] был классифицирован более узкий класс поверхностей Дель Пещц с нерациональньми особенностями.

Предполагается, что все рассматриваемые поверхности нормальные, комплексные и алгебраические. Автор очень признателен В. А. Исковских, Ю. Г. Прохорову за плодотворные обсуждения и интересные беседы.

\section{1. Линейчатые поверхности.}

Теорема 1. Пусть $\widetilde{X}-$ гладкая поверхность, $\tilde{\pi}: \tilde{X} \rightarrow C-$ сюрвективный морфизм на гладкую кривую $C$ такой, что каждый слой морфизма $\widetilde{\pi}$ изоморфен $\mathbb{P}^{1}$. Тогда

1) $\widetilde{X} \cong \mathbb{P}_{C}(\mathscr{E})$, әде $\mathscr{E}$ - локально свободный пучок ранга 2 такой, что $H^{0}(\mathscr{E}) \neq 0$ u $H^{0}(\mathscr{E} \otimes \mathscr{F})=0$ для любого $\mathscr{F} \in \operatorname{Pic}(\widetilde{X}), \operatorname{deg}(\mathscr{F})<0 ;$

2) $е=-\operatorname{deg}(\mathscr{E})$ является инвариантом поверхности $\widetilde{X}$;

3) существует сечение $C_{0}$ линейчатой поверхности $\widetilde{\pi}: \widetilde{X} \rightarrow C$ такое, что $C_{0}^{2}=-e$

4) $\operatorname{Pic}(\tilde{X}) \cong \mathbb{Z} C_{0} \oplus \widetilde{\pi}^{*} \operatorname{Pic}(C)$;

Работа выполнена при поддержке Международного научного фонда, грант № 9 О300. 
5) $K_{\tilde{X}} \sim-2 C_{0}+\tilde{\pi}^{*}\left(K_{C}+\wedge^{2} \mathscr{E}\right)$, в частности, $K_{\tilde{X}} \equiv-2 C_{0}+(2 g(C)-2-e) F$, где $F-$ слой морфизма $\tilde{\pi}$;

6) если е > $2 g(C)-2$, то пучок $\mathscr{E}$ разложим;

7) $C_{\lambda}^{2} \geqslant-е$ для любого сечения $C_{\lambda}$ линейчатой поверхности $\tilde{\pi}: \tilde{X} \rightarrow C$.

Доказательство см. в [3].

ОПРЕДЕЛЕниЕ 1. Поверхность $\widehat{X}$ назьвается линейчатой, если существует сюръективньй морфизм $\widehat{\pi}: \widehat{X} \rightarrow C$ на кривую $C$ такой, что общий слой морфизма $\widehat{\pi}$ изоморфен $\mathbb{P}^{1}$.

ЗАМЕчАниЕ 1 . В определении 1 кривая $C$ будет гладкой в силу нормальности поверхности $\widehat{X}$.

ОПредЕЛЕниЕ 2. Линейчатая поверхность $\tilde{\pi}: \widetilde{X} \rightarrow C$ назьвается относительно минимальной, если каждьй слой морфизма $\widetilde{\pi}$ неприводим, но возможно приведен.

Лемма 1. Для каждой линейчатой поверхности $\widehat{\pi}: \widehat{X} \rightarrow C$ существует коммутативная диаграмма

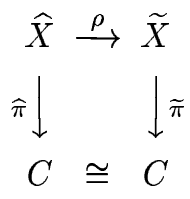

такая, что морфизм $\rho: \widehat{X} \rightarrow \widetilde{X}$ бирационален, $а \widetilde{\pi}: \widetilde{X} \rightarrow C$ есть относительно минимальная линейчатая поверхность.

ДокАЗАТЕЛЬСТво. Пусть $F-$ приводимый слой морфизма $\widehat{\pi}: \widehat{X} \rightarrow C$, тогда

$$
\left(\sum_{i=1}^{n} \lambda_{i} F_{i}\right)^{2} \leqslant 0, \quad\left(\sum_{i=1}^{n} \lambda_{i} F_{i}\right)^{2}=0 \Longleftrightarrow \sum_{i=1}^{n} \lambda_{i} F_{i}=\lambda F,
$$

где $F_{i}$ - компоненты слоя $F$ и $\lambda_{i}, \lambda \in \mathbb{Q}$ (см. [4]). Значит, для любого собственного подмножества $\left\{i_{1}, \ldots, i_{k}\right\} \subset\{1, \ldots, n\}$ форма пересечения дивизоров $F_{i_{j}}(j=1, \ldots, k)$ отрицательно определена, следовательно, дивизоры $F_{i_{j}}(j=1, \ldots, k)$ могут быть стянуты (см. [1]). Отсюда непосредственно получаем необходимый результат.

ЛЕмма 2. Относительно минимальной линейчатой поверхности $\widetilde{\pi}: \widetilde{X} \rightarrow C$ с сечением $C_{0}$ можсно канонически сопоставить гладкую относительно минимальную линейчатую поверхность $\widetilde{\pi}^{s}: \widetilde{X}^{s} \rightarrow C$ такую, что коммутативна диаграмма

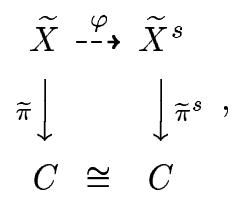

где 
ДокАЗАТЕЛЬСтво. Рассмотрим коммутативную диаграмму

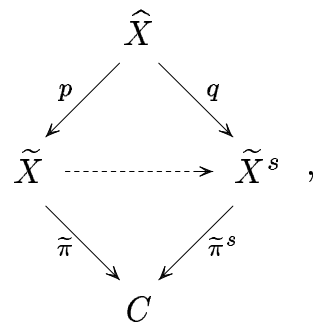

где $\widehat{X}$ - минимальное разрешение особенностей $\widetilde{X}$, а $\widetilde{X}^{s}$ - гладкая относительно минимальная над $C$ модель $\widehat{X}$. Нужно показать, что можно канонически выбрать морфизм $q$. В слоях морфизма $p$ нет $(-1)$-кривьг, но поверхность $\widetilde{\pi} \circ p: \widehat{X} \rightarrow C$ не относительно минимальная, следовательно, в каждом приводимом слое морфизма $\widetilde{\pi} \circ p$ лежит ровно одна (-1)-кривая, являющаяся собственным прообразом соответствующего слоя морфизма $\widetilde{\pi}$. Выберем $q$ так, чтобы $q=q_{1} \circ \cdots \circ q_{K}$ для некоторого $K \in \mathbb{N}_{\geqslant 0}$ (если $K=0$, то $\left.\widehat{X} \cong \widetilde{X} \cong \widetilde{X}^{s}\right)$, где

1) морфизм $q_{i}: \widehat{X}^{i} \rightarrow \widehat{X}^{i-1}\left(\widehat{X}^{K}=\widehat{X}\right.$ и $\left.\widehat{X}^{0}=\widetilde{X}^{s}\right)$ есть композиция раздутий в слое морфизма $\widetilde{\pi} \circ q_{1} \circ \cdots \circ q_{i-1}$ над точкой $x_{i} \in C$ для $i=1, \ldots, K$ и попарно различных $x_{i}$;

2) $q_{i}^{*}\left(q_{i} \circ \cdots \circ q_{K}\left(p^{-1}\left(C_{0}\right)\right)\right) \neq q_{i}^{-1}\left(q_{i} \circ \cdots \circ q_{K}\left(p^{-1}\left(C_{0}\right)\right)\right)$ для $i=1, \ldots, K$.

Легко видеть, что условиями 1$)-2$ ) морфизм $q$ определяется однозначно.

ЗАмЕЧАНИЕ 2. Из доказательства леммы 2 следует легкий алгоритм построения всех относительно минимальных линейчатых поверхностей. Достаточно взять гладкую относительно минимальную линейчатую поверхность и с некоторыми слоями сделать следующую перестройку:

1) раздуть точку на слое;

2) раздуть точку пересечения раздутой кривой и собственного прообраза слоя (двух $(-1)$-кривых);

3) последовательно делать раздутия точки на текущей $(-1)$-кривой так, чтобы в слое была только одна (-1)-кривая;

4) стянуть все кривые в слое за исключением единственной $(-1)$-кривой.

Заметим, что неоднозначность обратного перехода от особой поверхности к гладкой состоит в том, что при первом раздутии точки в слое неособой линейчатой поверхности появляются две $(-1)$-кривые.

ТЕОрема 2. Если $\widetilde{\pi}: \widetilde{X} \rightarrow C$-относительно минимальная линейчатая поверхность, mo

1) $\widetilde{X}-$ проективная поверхность;

2) $\widetilde{X}$ имеет не более чем рациональные особенности;

3) $R^{1} \widetilde{\pi}_{*}\left(\mathscr{O}_{\tilde{X}}\right)=0$;

4) все слои с приведенной структурой гладкие и изоморфны $\mathbb{P}^{1}$;

5) $\operatorname{rk}(\operatorname{Div}(\widetilde{X}) \otimes \mathbb{Q} / \equiv)=2$. 
ДокаЗАТЕЛЬСТво. 1) См. [5].

2) Рассмотрим коммутативную диаграмму (1), где морфизм $p$ - минимальное разрешение особенностей $\widetilde{X}$, а $q$ - бирациональньй морфизм на относительно минимальную гладкую линейчатую поверхность $\widetilde{\pi}^{s}: \widetilde{X}^{s} \rightarrow C$. Известно, что

$$
R^{1} \widetilde{\pi}_{*}^{s}\left(\mathscr{O}_{\tilde{X}^{s}}\right)=0, \quad R^{0} \widetilde{\pi}_{*}^{s}\left(\mathscr{O}_{\tilde{X}^{s}}\right)=\mathscr{O}_{C}
$$

и

$$
R^{1} q_{*}\left(\mathscr{O}_{\widehat{X}}\right)=0, \quad R^{0} q_{*}\left(\mathscr{O}_{\widehat{X}}\right)=\mathscr{O}_{\widetilde{X}^{s}} .
$$

Из спектральной последовательности Лере следует, что

$$
R^{1}(\widetilde{\pi} \circ p)_{*}\left(\mathscr{O}_{\widehat{X}}\right)=0, \quad R^{0}(\widetilde{\pi} \circ p)_{*}\left(\mathscr{O}_{\widehat{X}}\right)=\mathscr{O}_{C}
$$

Пусть $F=\sum_{i=1}^{n} a_{i} F_{i}$, где $F_{i}$ - неприводимые компоненты слоя $F$ и $a_{i} \in \mathbb{N}$. Тогда $R^{1}(\widetilde{\pi} \circ p)_{*}\left(\mathscr{O}_{\widehat{X}}\right)=0$ влечет $H^{1}\left(\mathscr{O}_{F}\right)=0$. Действительно, пусть $\mathscr{I}_{F}-$ пучок идеалов схемы $F$, тогда из точной последовательности

$$
0 \rightarrow \mathscr{I}_{F} \rightarrow \mathscr{O}_{\widehat{X}} \rightarrow \mathscr{O}_{F} \rightarrow 0
$$

следует точная последовательность

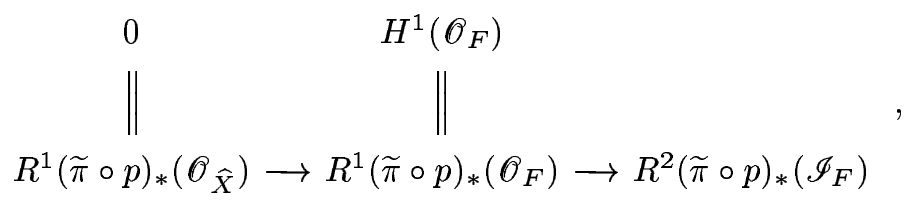

но $R^{2}(\widetilde{\pi} \circ p)_{*}\left(\mathscr{I}_{F}\right)=0$ из соображений размерности. Отсюда следует рациональность особенностей $\widetilde{X}$ (см. [6]) и рациональность особенностей любой поверхности, полученной из $\widehat{X}$ стягиванием компонент слоев морфизма $\widetilde{\pi} \circ p$.

3) Как доказано выше, особенности $\widetilde{X}$ рациональные, т.е. в обозначениях 2) имеем

$$
R^{1} p_{*}\left(\mathscr{O}_{\widehat{X}}\right)=0 \quad \text { и } \quad R^{0} p_{*}\left(\mathscr{O}_{\widehat{X}}\right)=\mathscr{O}_{\tilde{X}} .
$$

Спектральная последовательность Лере влечет

$$
R^{1} \widetilde{\pi}_{*}\left(\mathscr{O}_{\tilde{X}}\right)=0 \quad \text { и } \quad R^{0} \widetilde{\pi}_{*}\left(\mathscr{O}_{\tilde{X}}\right)=\mathscr{O}_{C} .
$$

4) Из рассуждений в 2$), 3)$ следует, что если $F$ - приведенньй слой морфизма $\widetilde{\pi}$, то $H^{1}\left(\mathscr{O}_{F}\right)=0$ и $F \cong \mathbb{P}^{1}$.

Заметим, что согласно замечанию 2 можно найти фундаментальные циклы (см. [6]) особенностей поверхности $\widetilde{X}$. Пересечение единственной $(-1)$-кривой в данном приводимом слоеморфизма $\widetilde{\pi} \circ p$ с соответствуюшими фундаментальньми циклами равно единище, откуда следует утверждение 4) (см. [6]).

5) См. [1].

ЗАМЕЧАНИЕ 3 . Из доказательства теоремы 2 следует, что особенности линейчатой поверхности рациональны. 


\section{2. Численные поверхности Дель Пеццо.}

ОПРЕДЕЛЕНИЕ 3. Дивизор Вейля $D$ на поверхности $X$ называется численно обильным, если для каждой кривой $C \in X$ вьполнено неравенство $D C>0$ и $D^{2}>0$.

ОПРЕДЕЛЕНИЕ 4. Поверхность $X$ назьвается численной поверхностью Дель Пеиио, если $-K_{X}$ есть численно обильньй дивизор Вейля.

Лемма 3. Пусть $Х$ есть численная поверхность Дель Пеццо, тогда

1) $H^{i}\left(\mathscr{O}_{X}\right)=0$ для $i=1,2$;

2) $Х$ есть проективная поверхность.

Доказательство 1) см. в [1], 2) - в[5].

Лемма 4. Пусть $X$ есть численная поверхность Дель Пецио, $f: \widehat{X} \rightarrow X-$ разрешение особенностей поверхности $X$. Тогда

1) $H^{1}\left(\mathscr{O}_{\widehat{X}}\right) \cong H^{0}\left(R^{1} f_{*}\left(\mathscr{O}_{\widehat{X}}\right)\right) u H^{2}\left(\mathscr{O}_{\widehat{X}}\right)=0$;

2) $\operatorname{kod}(\widehat{X})=-\infty$.

ДоказАТЕЛЬСТво. 1) Учитьвая лемму 3 , нормальность $X$ и спектральную последовательность Лере, получаем точную последовательность

$$
\begin{aligned}
& H^{1}\left(\mathscr{O}_{X}\right)=0 \\
& H^{2}\left(\mathscr{O}_{X}\right)=0 \\
& \| \\
& 0 \longrightarrow H^{1}\left(R^{0} f_{*}\left(\mathscr{O}_{\widehat{X}}\right)\right) \longrightarrow H^{1}\left(\mathscr{O}_{\widehat{X}}\right) \longrightarrow H^{0}\left(R^{1} f_{*}\left(\mathscr{O}_{\widehat{X}}\right)\right) \rightarrow H^{2}\left(R^{0} f_{*}\left(\mathscr{O}_{\widehat{X}}\right)\right) \\
& \rightarrow \quad H^{2}\left(\mathscr{O}_{\widehat{X}}\right) \quad \longrightarrow 0
\end{aligned}
$$

откуда следует необходимое утверждение.

2 ) Если существует эффективный дивизор $D \in\left|K_{\widehat{X}}\right|$, то $K_{X}=f_{*}(D)$, что невозможно, так как $-D$ есть численно обильньй дивизор на проективной поверхности (лемма 3 ).

СледСТВИЕ. Численная поверхность Дель Пецио рациональна тогда и только тогда, когда ее особенности рациональны.

\section{3. Численные поверхности Дель Пеццо с нерациональными особеннос- тями.}

Теорема 3. Пусть $X-$ численная поверхность Дель Пецио с нерациональными особенностями, a $f: \widehat{X} \rightarrow X$ - ее минимальное разрешение особенностей. Тогда

1) существует морфизм $\pi$ такой, что $\widehat{\pi}: \widehat{X} \rightarrow C$ есть линейчатая поверхность и $g(C)=H^{1}\left(\mathscr{O}_{\widehat{X}}\right) \neq 0$;

2) морфизм $f$ стягивает одну гладкую кривую $E$, не лехсащую в слоях морфизма $\widehat{\pi}$, более того, Е является сечением морфизма $\widehat{\pi} ;$

3) для любой относительно минимальной над $C$ модели $\widetilde{\pi}^{s}: \widetilde{X}^{s} \rightarrow C$ линейчатой поверхности $\widehat{X}$ выполнено

$$
\widehat{\pi}=\widetilde{\pi}^{s} \circ \rho, \quad \widetilde{X}^{s} \cong \mathbb{P}_{C}(\mathscr{E}), \quad e>2 g(C)-2, \quad \rho(E)^{2}=-e,
$$

әде $\mathscr{E}-$ разложимый локально свободный пучок ранга 2 , ае - инвариант $\mathbb{P}_{C}(\mathscr{E})$. 
ДокАЗАТЕльство. 1) Утверждение непосредственное вытекает из леммы 4 и следствия.

2) Заметим, что морфизм $f$ должен стягивать хотя бы одну кривую, не лежащую в слоях морфизма $\widehat{\pi}$, так как иначе по замечанию 3 особенности $X$ были бы рациональные. Пусть $E_{j}(j=1, \ldots, k)$ - неприводимые приведенные кривые, не лежащие в слоях морфизма $\widehat{\pi}$ и стягиваемые морфизмом $f$. Тогда

$$
K_{\widehat{X}} \equiv f^{*}\left(K_{X}\right)-\sum_{i=1}^{n} a_{i} F_{i}-\sum_{j=1}^{k} b_{j} E_{j}
$$

где $F_{i}$ - исключительные кривые морфизма $f$, лежащие в слоях морфизма $\widehat{\pi} ; a_{i}, b_{j} \in \mathbb{Q} \geqslant 0$ для $i=1, \ldots, n$ и $j=1, \ldots, k$. По формуле присоединения для кривой $E_{r}(r \in\{1, \ldots, k\})$

$$
\left(K_{\widehat{X}}+E_{r}\right) E_{r} \geqslant 2 g\left(\widetilde{E}_{r}\right)-2,
$$

где $\widetilde{E}_{r}-$ нормализация кривой $\widetilde{E}_{r}$. По формуле Гурвица

$$
2 g\left(\widetilde{E}_{r}\right)-2 \geqslant 2 g(C)-2 \geqslant 0,
$$

и следовательно,

$$
\left(1-b_{r}\right) E_{r}^{2} \geqslant\left(-\sum_{i=1}^{n} a_{i} F_{i}-\sum_{j=1, j \neq r}^{k} b_{j} E_{j}-\left(b_{r}-1\right) E_{r}\right) E_{r} \geqslant 0 .
$$

Таким образом, все $b_{j} \geqslant 1$. Если $L$ есть слой морфизма $\widehat{\pi}$, то

$$
-2=K_{\widehat{X}} L=\left(f^{*}\left(K_{X}\right)-\sum_{i=1}^{n} a_{i} F_{i}-\sum_{j=1}^{k} b_{j} E_{j}\right) L<\left(-\sum_{j=1}^{k} b_{j} E_{j}\right) L
$$

следовательно, $k=1, b=b_{1}<2, E=E_{1} \cong \widetilde{E}_{1}$ есть сечение линейчатой поверхности $\widehat{\pi}: \widehat{X} \rightarrow C$.

3) Возьмем сечение $C_{0}$ линейчатой поверхности $\widetilde{\pi}^{s}: \widetilde{X}^{s} \rightarrow C$ такое, что $C_{0}^{2}=-e$. Тогда

$$
\rho(E) \equiv C_{0}+d F
$$

где $F$ есть слой морфизма $\tilde{\pi}^{s}$ и $d \in \mathbb{N}$ по теореме 1 . В обозначениях 2) имеем

$$
\rho\left(\sum_{i=1}^{n} a_{i} F_{i}\right) \equiv a F
$$

и

$$
K_{\tilde{X}^{s}}+\rho\left(\sum_{i=1}^{n} a_{i} F_{i}+b E\right) \equiv(b-2) C_{0}+(2 g(C)-2-e+a+d b) F
$$


где $a \in \mathbb{Q} \geqslant 0$. Если $C_{0} \neq \rho(E)$, то $\rho(E) C_{0}=d-e \geqslant 0$ и

$$
\begin{aligned}
b d-b e+2 g(C)-2+e+a & =\left(K_{\tilde{X}^{s}}+\rho\left(\sum_{i=1}^{n} a_{i} F_{i}+b E\right)\right) C_{0} \\
& =\left(K_{\widehat{X}}+\sum_{i=1}^{n} a_{i} F_{i}+b E\right) \rho^{*}\left(C_{0}\right)=f^{*}\left(K_{X}\right) \rho^{*}\left(C_{0}\right)<0 .
\end{aligned}
$$

Но если $e \geqslant 0$, то

$$
b d-b e+2 g(C)-2+e+a>b(d-e) \geqslant 0 .
$$

Если $e<0$, то

$$
b d-b e+2 g(C)-2+e+a>e(1-b) \geqslant 0 .
$$

Значит, $C_{0}=\rho(E)$. Аналогично,

$$
\begin{aligned}
b e+2 g(C)-2+e+a & =\left(K_{\tilde{X}^{s}}+\rho\left(\sum_{i=1}^{n} a_{i} F_{i}+b E\right)\right) C_{0} \\
& =\left(K_{\widehat{X}}+\sum_{i=1}^{n} a_{i} F_{i}+b E\right) \rho^{*}\left(C_{0}\right)=f^{*}\left(K_{X}\right) \rho^{*}\left(C_{0}\right) .
\end{aligned}
$$

Заметим, что если $\rho^{-1}\left(C_{0}\right) \neq \rho^{*}\left(C_{0}\right)$, то $f^{*}\left(K_{X}\right) \rho^{*}\left(C_{0}\right)<0$, поскольку в этом случае $\rho^{*}\left(C_{0}\right)$ содержит $(-1)$-кривую, которая не может быть стянута морфизмом $f$. Если $C_{0}^{2}=-e \geqslant 0$, то $\rho^{-1}\left(C_{0}\right) \neq \rho^{*}\left(C_{0}\right)$ и

$$
0>f^{*}\left(K_{X}\right) \rho^{*}\left(C_{0}\right)=(1-b) e+2 g(C)-2+a \geqslant 0 .
$$

Следовательно, $e>0$ и

$$
0>f^{*}\left(K_{X}\right) \rho^{*}\left(C_{0}\right)=(1-b) e+2 g(C)-2+a \geqslant-e+2 g(C)-2,
$$

откуда по теореме 1 получаем, что пучок $\mathscr{E}$ разложим.

Tеорема 4. В условиях теоремы 3 пусть $\operatorname{rk}(\operatorname{Div}(X) \otimes \mathbb{Q} / \equiv)=1$. Тогда $X$ есть стягивание сечения относительно минимальной линейчатой поверхности $\tilde{\pi}: \widetilde{X} \rightarrow C u h^{1}\left(\mathscr{O}_{\tilde{X}}\right)=g(C)>0$. Более того, поверхность $\widetilde{X}$ определяется поверхностью $X$ единственным образом.

ДокаЗАТЕЛЬство. Пусть $f: \widehat{X} \rightarrow X$ есть минимальное разрешение особенностей $X$. Тогда по теореме $3 \widehat{X}$ есть линейчатая поверхность $\widehat{\pi}: \widehat{X} \rightarrow C$ такая, что $g(C)>0, f$ стягивает одно сечение и компоненты приводимых слоев морфизма $\widehat{\pi}$. Пусть

$$
F^{\lambda}=\sum_{i=1}^{j_{\lambda}} a_{i} F_{i}^{\lambda} \quad\left(\lambda=1, \ldots, N, \quad a_{i} \in \mathbb{N}\right)
$$

- приводимые слои морфизма $\widehat{\pi}$. Тогда

$$
\operatorname{rk}(\operatorname{Div}(\widehat{X}) \otimes \mathbb{Q} / \equiv)=2+\sum_{\lambda=1}^{N}\left(j_{\lambda}-1\right) .
$$


С другой стороны,

$$
\operatorname{rk}(\operatorname{Div}(\widehat{X}) \otimes \mathbb{Q} / \equiv)=1+\text { число кривых, стягиваемых морфизмом } f .
$$

Следовательно, морфизм $f$ не стягивает только одну компоненту каждого приводимого слоя, и существует следующая коммутативная диаграмма:

$$
\begin{aligned}
& \widehat{X} \stackrel{p}{\longrightarrow} \tilde{X} \stackrel{g}{\longrightarrow} X \\
& \widehat{\pi} \downarrow \quad \downarrow \tilde{\pi} \quad, \\
& C \cong C
\end{aligned}
$$

где $f=g \circ p, \tilde{\pi}: \widetilde{X} \rightarrow C$ - относительно минимальная линейчатая поверхность, и морфизм $g$ стягивает ее сечение.

Так как $h^{1}\left(\mathscr{O}_{\widehat{X}}\right)=g(C)$ и по теореме 2 особенности $\widetilde{X}$ рациональны, то из спектральной последовательности Лере следует, что $h^{1}\left(\mathscr{O}_{\tilde{X}}\right)=g(C)>0$.

Единственность поверхности $\widetilde{X}$ следует из ее построения.

Теорема 5. При выполнении условий теоремы 4 поверхности $X$ можно канонически сопоставить гладкую относительно минимальную линейчатую поверхность $\widetilde{\pi}^{s}: \tilde{X}^{s} \rightarrow C$ такую, что $\widetilde{X}^{s} \cong \mathbb{P}_{C}(\mathscr{E})$, где $\mathscr{E}-$ локально свободный пучок ранга $2, e>2 g(C)-2$, пучок $\mathscr{E}$ разложим и $q\left(p^{-1}(E)\right)^{2}=-e$, где е - инвариант линейчатой поверхности $\mathbb{P}_{C}(\mathscr{E})$.

Доказательство теоремы следует из теорем 3,4 и леммы 2.

4. Конструкция. Пусть дана пара $\left(\widehat{\pi}: \widehat{X} \rightarrow C, C_{0}\right)$, где $\widehat{\pi}: \widehat{X} \rightarrow C$-гладкая линейчатая поверхность и $C_{0}$ - ее сечение. Скажем, что пара $\left(\widehat{\pi}^{\prime}: \widehat{X}^{\prime} \rightarrow C, C_{0}^{\prime}\right)$ получена әлементарным преобразованием $\varphi$ над точкой $x \in C$ из пары $\left(\widehat{\pi}: \widehat{X} \rightarrow C, C_{0}\right)$, если дана коммутативная диаграмма

такая, что

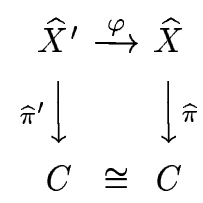

1) $\widehat{\pi}^{\prime}: \widehat{X}^{\prime} \rightarrow C$-гладкая линейчатая поверхность;

2) $\varphi$ - бирациональньй морфизм, причем $\varphi$ есть композиция раздутий в слое морфизма $\widehat{\pi}$ над точкой $x \in C$;

3) слой морфизма $\widehat{\pi}$ над точкой $x \in C$ неприводим;

4) в слое морфизма $\widehat{\pi}^{\prime}$ над $x$ лежит ровно одна $(-1)$-кривая;

5) $C_{0}^{\prime}=\varphi^{-1}\left(C_{0}\right)$ и $\varphi^{*}\left(C_{0}\right) \neq C_{0}^{\prime}$.

ОПрЕДЕЛЕниЕ 5. Последовательность пар целых чисел $\left(\alpha_{i}^{1}, \alpha_{i}^{2}\right)$ для $i \in \mathbb{N}_{\geqslant 3}$ обладает свойством $(*)$, если

1) $\left(\alpha_{3}^{1}, \alpha_{3}^{2}\right)=(1,1)$

2 ) для $i \geqslant 4$

$$
\left(\alpha_{i}^{1}, \alpha_{i}^{2}\right)= \begin{cases}\left(\alpha_{i-1}^{1}, \alpha_{i-1}^{1}+\alpha_{i-1}^{2}\right), & \text { либо } \\ \left(\alpha_{i-1}^{1}+\alpha_{i-1}^{2}, \alpha_{i-1}^{2}\right), & \text { либо } \\ \left(0, \alpha_{i-1}^{1}+\alpha_{i-1}^{2}\right) . & \end{cases}
$$


Пусть пара $\left(\widehat{\pi}^{\prime}: \widehat{X}^{\prime} \rightarrow C, C_{0}^{\prime}\right)$ получена элементарньм преобразованием $\varphi$ над точкой $x \in C$ из пары $\left(\widehat{\pi}: \widehat{X} \rightarrow C, C_{0}\right)$. Введем следующие обозначения:

1) $\widehat{X}_{0}=\widehat{X}, \widehat{\pi}_{0}=\widehat{\pi}, F_{1}-$ слой морфизма $\widehat{\pi}_{0}$ над точкой $x$;

2) $\chi_{1,0}: \widehat{X}_{1} \rightarrow \widehat{X}_{0}-$ раздутие точки $F_{1} \cap C_{0}, \widehat{\pi}_{1}=\widehat{\pi}_{0} \circ \chi_{1,0}, F_{2}$ - исключительная кривая морфизма $\chi_{1,0}$

3) $\chi_{2,1}: \widehat{X}_{2} \rightarrow \widehat{X}_{1}$ - раздутие точки $F_{1} \cap F_{2}, \chi_{2,0}=\chi_{1,0} \circ \chi_{2,1}, \widehat{\pi}_{2}=\widehat{\pi}_{1} \circ \chi_{2,1}, F_{3}-$ исключительная кривая морфизма $\chi_{2,1}$;

4) $\chi_{i+1, i}: \widehat{X}_{i+1} \rightarrow \widehat{X}_{i}$ - раздутие точки на $F_{i+1}, \chi_{i+1, j}=\chi_{j+1, j} \circ \cdots \circ \chi_{i+1, i}$, если $j \leqslant i, \widehat{\pi}_{i+1}=\widehat{\pi}_{i} \circ \chi_{i+1, i}, F_{i+2}-$ исключительная кривая морфизма $\chi_{i+1, i}$;

5) $F^{r}$ - возможно неприведенньй слой морфизма $\widehat{\pi}_{r}$ над точкой $x$;

6) $\widehat{X}_{N}=\widehat{X}^{\prime}, \widehat{\pi}_{N}=\widehat{\pi}^{\prime}, F_{N+1}$ - единственная $(-1)$-кривая в $F^{N}$;

7) $C_{0}^{\prime}=\chi_{N, 0}^{-1}\left(C_{0}\right)$ и $\bar{F}_{i}=\chi_{N, i-1}^{-1}\left(F_{i}\right)$ для $i=1, \ldots, N-1$.

Сопоставим поверхности $\widehat{X}^{\prime}$ последовательность пар целых чисел

$$
\left(\bar{\alpha}_{i}^{1}\left(\widehat{X}^{\prime}\right), \bar{\alpha}_{i}^{2}\left(\widehat{X}^{\prime}\right)\right) \quad(i=3, \ldots, N+1)
$$

где $N+1$ - число неприводимых компонент в слое морфизма $\widehat{\pi}^{\prime}$ над точкой $x \in C$. Для $i=1, \ldots, N-1$ рассмотрим поверхность $\widehat{X}_{i+1}$, тогда

$$
F^{i+1} \equiv a_{i+2} F_{i+2}+\sum_{j=1}^{i+1} a_{j} \chi_{i+1, j-1}^{-1}\left(F_{j}\right)
$$

$F_{i+2}$ - единственная $(-1)$-кривая в $F^{i+1}$, причем $F_{i+2}$ пересекает не более двух неприводимых компонент $F^{i+1}$. Пусть $F_{i+2}$ пересекает $\chi_{i+1, k-1}^{-1}\left(F_{k}\right)$ и $\chi_{i+1, l-1}^{-1}\left(F_{l}\right)$, причем $\chi_{i+1, l-1}^{-1}\left(F_{l}\right)$ лежит в связной компоненте $F^{i+1} \backslash F_{i+2}$, которая пересекается с $\chi_{i+1,0}^{-1}\left(C_{0}\right)$, где $l \neq k$ и $k, l \in\{1, \ldots, i+1\}$. Положим

$$
\left(\bar{\alpha}_{i+2}^{1}\left(\widehat{X}^{\prime}\right), \bar{\alpha}_{i+2}^{2}\left(\widehat{X}^{\prime}\right)\right)=\left(a_{k}, a_{l}\right) .
$$

Пусть $F_{i+2}$ пересекает только $\chi_{i+1, k-1}^{-1}\left(F_{k}\right)$ среди компонент $F^{i+1}(k \in\{1, \ldots, i+1\})$, тогда $k=i+1$. В этом случае положим

$$
\left(\bar{\alpha}_{i+2}^{1}\left(\widehat{X}^{\prime}\right), \bar{\alpha}_{i+2}^{2}\left(\widehat{X}^{\prime}\right)\right)=\left(0, a_{i+1}\right) .
$$

ЛЕмма 5. Последовательность пар целых чисел (2) удовлетворяет условию (*). ДоКАЗАТЕЛЬСТво. Обозначим

$$
\left(\bar{\alpha}_{i}^{1}\left(\widehat{X}^{\prime}\right), \bar{\alpha}_{i}^{2}\left(\widehat{X}^{\prime}\right)\right)=\left(\bar{\alpha}_{i}^{1}, \bar{\alpha}_{i}^{2}\right) \quad(i=3, \ldots, N+1) .
$$

На поверхности $\widehat{X}_{2}$ выполнено соотношение

$$
F^{2} \equiv 2 F_{3}+\chi_{2,1}^{-1}\left(F_{2}\right)+\chi_{2,0}^{-1}\left(F_{1}\right)
$$

Непосредственно проверяется, что $\left(\bar{\alpha}_{3}^{1}, \bar{\alpha}_{3}^{2}\right)=(1,1)$.

Пусть последовательность пар целых чисел $\left(\bar{\alpha}_{i}^{1}, \bar{\alpha}_{i}^{2}\right)$ удовлетворяет условию $(*)$ для $i=3, \ldots, r$. Докажем, что она удовлетворяет условию $(*)$ для $i=3, \ldots, r+1$. 
На поверхностях $\widehat{X}_{r-1}$ и $\widehat{X}_{r}$ выполнены соотношения

$$
\begin{aligned}
F^{r-1} & \equiv a_{r} F_{r}+\sum_{j=1}^{r-1} a_{j} \chi_{r-1, j-1}^{-1}\left(F_{j}\right) \\
F^{r} & \equiv a_{r+1} F_{r+1}+\sum_{j=1}^{r} a_{j} \chi_{r, j-1}^{-1}\left(F_{j}\right) .
\end{aligned}
$$

Пусть $F_{r}$ пересекает $\chi_{r-1, k-1}^{-1}\left(F_{k}\right)$ и $\chi_{r-1, l-1}^{-1}\left(F_{l}\right)$, причем $\chi_{r-1, l-1}^{-1}\left(F_{l}\right)$ лежит в связной компоненте $F^{r-1} \backslash F_{r}$, которая пересекается с $\chi_{r-1,0}^{-1}\left(C_{0}\right)$, где $l \neq k$ и $k, l \in\{1, \ldots$, $r-1\}$. По предположению $\left(\bar{\alpha}_{r}^{1}, \bar{\alpha}_{r}^{2}\right)=\left(a_{k}, a_{l}\right)$. Разберем три случая.

1) Пусть $\chi_{r, r-1}: \widehat{X}_{r} \rightarrow \widehat{X}_{r-1}$ есть раздутие $F_{r} \cap \chi_{r-1, k-1}^{-1}\left(F_{k}\right)$. В этом случае $F_{r+1}$ пересекает $\chi_{r, r-1}^{-1}\left(F_{r}\right)$ и $\chi_{r, k-1}^{-1}\left(F_{k}\right)$, причем $\chi_{r, r-1}^{-1}\left(F_{r}\right)$ лежит в связной компоненте $F^{r} \backslash F_{r+1}$, которая пересекается с $\chi_{r, 0}^{-1}\left(C_{0}\right)$. По определению $\left(\bar{\alpha}_{r+1}^{1}, \bar{\alpha}_{r+1}^{2}\right)=\left(a_{k}, a_{r}\right)$, где $a_{r}=a_{k}+a_{l}$.

2) Пусть $\chi_{r, r-1}: \widehat{X}_{r} \rightarrow \widehat{X}_{r-1}$ есть раздутие $F_{r} \cap \chi_{r-1, l-1}^{-1}\left(F_{l}\right)$. В этом случае $F_{r+1}$ пересекает $\chi_{r, r-1}^{-1}\left(F_{r}\right)$ и $\chi_{r, k-1}^{-1}\left(F_{k}\right)$, причем $\chi_{r, k-1}^{-1}\left(F_{k}\right)$ лежит в связной компоненте $F^{r} \backslash F_{r+1}$, которая пересекается с $\chi_{r, 0}^{-1}\left(C_{0}\right)$. По определению $\left(\bar{\alpha}_{r+1}^{1}, \bar{\alpha}_{r+1}^{2}\right)=\left(a_{r}, a_{l}\right)$, где $a_{r}=a_{k}+a_{l}$.

3) Пусть, наконец, $\chi_{r, r-1}: \widehat{X}_{r} \rightarrow \widehat{X}_{r-1}$ есть раздутие точки на $F_{r}$, не принадлежащей $\chi_{r-1, k-1}^{-1}\left(F_{k}\right) \cup \chi_{r-1, l-1}^{-1}\left(F_{l}\right)$. В этом случае $F_{r+1}$ пересекает только $\chi_{r, r-1}^{-1}\left(F_{r}\right)$ среди компонент $F^{r}$. По определению $\left(\bar{\alpha}_{r+1}^{1}, \bar{\alpha}_{r+1}^{2}\right)=\left(0, a_{r}\right)$, где $a_{r}=a_{k}+a_{l}$.

Пусть $F_{r}$ пересекает только $\chi_{r-1, r-2}^{-1}\left(F_{r-1}\right)$ среди компонент $F^{r-1}$. По предположению $\left(\bar{\alpha}_{r}^{1}, \bar{\alpha}_{r}^{2}\right)=\left(0, a_{r-1}\right)$. Заметим, что $\chi_{r, r-1}: \widehat{X}_{r} \rightarrow \widehat{X}_{r-1}$ есть раздутие либо $F_{r} \cap \chi_{r-1, r-2}^{-1}\left(F_{r-1}\right)$, либо точки на $F_{r}$, не принадлежащей $\chi_{r-1, r-2}^{-1}\left(F_{r-1}\right)$. Разберем два случая.

1) Пусть $\chi_{r, r-1}: \widehat{X}_{r} \rightarrow \widehat{X}_{r-1}$ есть раздутие $F_{r} \cap \chi_{r-1, r-2}^{-1}\left(F_{r-1}\right)$. В этом случае $F_{r+1}$ пересекает $\chi_{r, r-1}^{-1}\left(F_{r}\right)$ и $\chi_{r, r-2}^{-1}\left(F_{r-1}\right)$, причем $\chi_{r, r-2}^{-1}\left(F_{r-1}\right)$ лежит в связной компоненте $F^{r} \backslash F_{r+1}$, которая пересекается с $\chi_{r, 0}^{-1}\left(C_{0}\right)$. По определению $\left(\bar{\alpha}_{r+1}^{1}, \bar{\alpha}_{r+1}^{2}\right)=\left(a_{r}, a_{r-1}\right)$, где $a_{r}=a_{r-1}$.

2) Пусть теперь $\chi_{r, r-1}: \widehat{X}_{r} \rightarrow \widehat{X}_{r-1}$ есть раздутие точки на $F_{r}$, не принадлежащей $\chi_{r-1, r-2}^{-1}\left(F_{r-1}\right)$. В этом случае $F_{r+1}$ пересекает только $\chi_{r, r-1}^{-1}\left(F_{r}\right)$ среди компонент $F^{r}$. По определению $\left(\bar{\alpha}_{r+1}^{1}, \bar{\alpha}_{r+1}^{2}\right)=\left(0, a_{r}\right)$, где $a_{r}=a_{r-1}$.

Во всех случаях последовательность пар целых чисел $\left(\bar{\alpha}_{i}^{1}, \bar{\alpha}_{i}^{2}\right)$ удовлетворяет условию $(*)$ для $i=3, \ldots, r+1$. Лемма доказана.

Лемма 6. Пусть последовательность пар иельх чисел $\left(\alpha_{i}^{1}, \alpha_{i}^{2}\right)$, удовлетворяет условию (*). Тогда существует единственная пара $\left(\widehat{\pi}^{\prime}: \widehat{X}^{\prime} \rightarrow C, C_{0}^{\prime}\right)$, полученная әлементарным преобразованием $\varphi$ над точкой $x \in C$ из пары $\left(\widehat{\pi}: \widehat{X} \rightarrow C, C_{0}\right)$, такая, что выполнены соотношения (3).

ДокАЗАТЕЛЬСтво. Индуктивно найдем всеповерхности $\widehat{X}_{r}$. Допустим, что мы уже нашли поверхность $\widehat{X}_{r}$ для некоторого $r \in\{2, \ldots, N\}$. Найдем поверхность $\widehat{X}_{r+1}$.

Пусть $F_{r+1}$ пересекает $\chi_{r, k-1}^{-1}\left(F_{k}\right)$ и $\chi_{r, l-1}^{-1}\left(F_{l}\right)$, причем $\chi_{r, l-1}^{-1}\left(F_{l}\right)$ лежит в связной компоненте $F^{r} \backslash F_{r+1}$, которая пересекается с $\chi_{r, 0}^{-1}\left(C_{0}\right)$, где $l \neq k$ и $k, l \in\{1, \ldots, r\}$. Разберем три случая. 
1) Пусть $\left(\alpha_{r+1}^{1}, \alpha_{r+1}^{2}\right)=\left(0, \alpha_{r}^{1}+\alpha_{r}^{2}\right)$. В этом случае $\chi_{r+1, r}: \widehat{X}_{r+1} \rightarrow \widehat{X}_{r}$ есть раздутие точки на $F_{r+1}$, которая не принадлежит $\chi_{r, l-1}^{-1}\left(F_{l}\right) \cup \chi_{r, l-1}^{-1}\left(F_{k}\right)$.

2) Пусть $\left(\alpha_{r+1}^{1}, \alpha_{r+1}^{2}\right)=\left(\alpha_{r}^{1}, \alpha_{r}^{2}+\alpha_{r}^{1}\right)$. В этом случае $\chi_{r+1, r}: \widehat{X}_{r+1} \rightarrow \widehat{X}_{r}$ есть раздутие точки на $F_{r+1} \cap \chi_{r, k-1}^{-1}\left(F_{k}\right)$.

3) Наконец, $\left(\alpha_{r+1}^{1}, \alpha_{r+1}^{2}\right)=\left(\alpha_{r}^{1}+\alpha_{r}^{2}, \alpha_{r}^{2}\right)$. В этом случае $\chi_{r+1, r}: \widehat{X}_{r+1} \rightarrow \widehat{X}_{r}$ есть раздутие точки на $F_{r+1} \cap \chi_{r, l-1}^{-1}\left(F_{l}\right)$.

Пусть $F_{r+1}$ пересекает только $\chi_{i+1, r-1}^{-1}\left(F_{r}\right)$ среди всех неприводимых компонент $F^{r}$. Разберем два случая.

1) Если $\left(\alpha_{r+1}^{1}, \alpha_{r+1}^{2}\right)=\left(0, \alpha_{r}^{2}\right)$, то $\chi_{r+1, r}: \widehat{X}_{r+1} \rightarrow \widehat{X}_{r}$ есть раздутие точки на $F_{r+1}$, которая не принадлежит $\chi_{r, r-1}^{-1}\left(F_{r}\right)$.

2) Если $\left(\alpha_{r+1}^{1}, \alpha_{r+1}^{2}\right)=\left(\alpha_{r}^{2}, \alpha_{r}^{2}\right)$, то $\chi_{r+1, r}: \widehat{X}_{r+1} \rightarrow \widehat{X}_{r}$ есть раздутие точки на $F_{r+1} \cap \chi_{r, r-1}^{-1}\left(F_{r}\right)$.

Легко видеть, что полученная таким образом поверхность $\widehat{X}_{N}=\widehat{X}^{\prime}$ единственна и соотношения (3) для нее вьполнены.

ОПРЕДЕлЕниЕ 6. Последовательность пар целых чисел $\left(\beta_{i}^{1}, \beta_{i}^{2}\right)\left(i \in \mathbb{N}_{\geqslant 3}\right)$ двойственна к последовательности пар целых чисел $\left(\alpha_{i}^{1}, \alpha_{i}^{2}\right)\left(i \in \mathbb{N}_{\geqslant 3}\right)$, удовлетворяющей условию $(*)$, если

1) $\left(\beta_{3}^{1}, \beta_{3}^{2}\right)=(0,-1)$;

2) для $i \geqslant 4$

$$
\left(\beta_{i}^{1}, \beta_{i}^{2}\right)= \begin{cases}\left(\beta_{i-1}^{1}, \beta_{i-1}^{1}+\beta_{i-1}^{2}+1\right), & \text { если }\left(\alpha_{i}^{1}, \alpha_{i}^{2}\right)=\left(\alpha_{i-1}^{1}, \alpha_{i-1}^{1}+\alpha_{i-1}^{2}\right) ; \\ \left(\beta_{i-1}^{1}+\beta_{i-1}^{2}+1, \beta_{i-1}^{2}\right), & \text { если }\left(\alpha_{i}^{1}, \alpha_{i}^{2}\right)=\left(\alpha_{i-1}^{1}+\alpha_{i-1}^{2}, \alpha_{i-1}^{2}\right) ; \\ \left(0, \beta_{i-1}^{1}+\beta_{i-1}^{2}+1\right), & \text { если }\left(\alpha_{i}^{1}, \alpha_{i}^{2}\right)=\left(0, \alpha_{i-1}^{1}+\alpha_{i-1}^{2}\right) .\end{cases}
$$

\section{5. Классификация. Пусть даны}

1) гладкая относительно минимальная линейчатая поверхность $\pi^{0}: X^{0} \rightarrow C$ с инвариантом $e$ такая, что $X^{0} \cong \mathbb{P}_{C}\left(\mathscr{O}_{C} \oplus \mathscr{L}\right)$, где $\mathscr{L} \in \operatorname{Pic}(C), C_{0}$ - единственное сечение этой линейчатой поверхности с $C_{0}^{2}=-e$ и $e=-\operatorname{deg}(\mathscr{L})>2 g(C)-2$;

2) набор попарно различных точек $\left\{x_{1}, \ldots, x_{K}\right\} \subset C$, возможно пустой $(K=0)$;

3) гладкие линейчатые поверхности $\pi^{d}: X^{d} \rightarrow C$ с сечениями $C_{d} \subset X^{d}$ для $d=1$, $\ldots, K$ такие, что пара $\left(\pi^{d}: X^{d} \rightarrow C, C_{d}\right)$ получена элементарньм преобразованием $\varphi_{d}$ над точкой $x_{d} \in C$ из пары $\left(\pi^{d-1}: X^{d-1} \rightarrow C, C_{d-1}\right)$ для $d=1, \ldots, K$.

Если $K \geqslant 1$, то конструкция из предыдущего пункта ставит в соответствие каждой поверхности $X^{d}(d=1, \ldots, K)$ последовательность пар целых чисел

$$
\left(\bar{\alpha}_{i}^{1}\left(X^{d}\right), \bar{\alpha}_{i}^{2}\left(X^{d}\right)\right) \quad(i=3, \ldots, N(d)+1)
$$

и двойственную к ней последовательность пар целых чисел

$$
\left(\bar{\beta}_{i}^{1}\left(X^{d}\right), \bar{\beta}_{i}^{2}\left(X^{d}\right)\right) \quad(i=3, \ldots, N(d)+1),
$$

где $N(d)+1$ - число неприводимых компонент слоя морфизма $\pi^{d}$ над точкой $x_{d}$. Поверхности $X_{K}$ сопоставляется $2 K$ последовательностей пар целых чисел

$$
\left(\bar{\alpha}_{i}^{1}\left(X^{K}, d\right), \bar{\alpha}_{i}^{2}\left(X^{K}, d\right)\right)=\left(\bar{\alpha}_{i}^{1}\left(X^{d}\right), \bar{\alpha}_{i}^{2}\left(X^{d}\right)\right)
$$


и

$$
\left(\bar{\beta}_{i}^{1}\left(X^{K}, d\right), \bar{\beta}_{i}^{2}\left(X^{K}, d\right)\right)=\left(\bar{\beta}_{i}^{1}\left(X^{d}\right), \bar{\beta}_{i}^{2}\left(X^{d}\right)\right)
$$

для $d=1, \ldots, K$ и $i=3, \ldots, N(d)+1$, где $N(d)+1$ - число неприводимых компонент слоя морфизма $\pi^{K}$ над точкой $x_{d}$.

ЛЕмМА 7. В обозначениях данного пункта

1) последовательность пар иелых чисел

$$
\left(\bar{\alpha}_{i}^{1}\left(X^{K}, d\right), \bar{\alpha}_{i}^{2}\left(X^{K}, d\right)\right) \quad(d=1, \ldots, K, \quad i=3, \ldots, N(d)+1)
$$

удовлетворяют условию (*) при фиксированном $d$;

2) для заданных $K$ последовательностей пар иелых чисел

$$
\left(\alpha_{i}^{1}(d), \alpha_{i}^{2}(d)\right) \quad\left(d=1, \ldots, K, \quad i=3, \ldots, R(d)+1, \quad R(d) \in \mathbb{N}_{\geqslant 2}\right),
$$

удовлетворяющих условию (*), существует единственная гладкая линейчатая поверхность $\pi^{K}: X^{K} \rightarrow C$ с сечением $C_{K}$ такая, что пара $\left(\pi^{K}\right.$ : $\left.X^{K} \rightarrow C, C_{K}\right)$ получена из пары $\left(\pi^{0}: X^{0} \rightarrow C, C_{0}\right)$ с помощью последовательности әлементарных преобразований над точками $\left\{x_{1}, \ldots, x_{K}\right\} \subset C$,

$$
\left(\bar{\alpha}_{i}^{1}\left(X^{K}, d\right), \bar{\alpha}_{i}^{2}\left(X^{K}, d\right)\right)=\left(\alpha_{i}^{1}(d), \alpha_{i}^{2}(d)\right) \quad(d=1, \ldots, K, \quad i=3, \ldots, R(d)+1),
$$

где $R(d)+1$ - число неприводимых компонент в слое морфизма $\pi^{K}$ над мочкой $x_{d}$.

Доказательство следует из лемм 5, 6.

Лемма 8. Пусть в обозначениях данного пункта $F(d)$ - возможсно неприведенный слой морфизма $\pi^{K}$ над точкой $x_{d}(d=1 \ldots, K, K \geqslant 0)$, и на поверхности $X^{K}$ выполнены соотношения

$$
F(d) \equiv \sum_{j=1}^{N(d)+1} a_{j}(d) \bar{F}_{j}(d)
$$

$u$

$$
K_{X^{K}} \equiv-2 C_{K}+\sum_{d=1}^{K} \sum_{j=1}^{N(d)+1} b_{j}(d) \bar{F}_{j}(d)+(2 g(C)-2-e) F
$$

где $\bar{F}_{j}(d)$ - неприводимые компоненты слоя морфизма $\pi^{K}$ над точкой $x_{d}$ в обозначениях $n$. 4, а F- общий слой морфизма $\pi^{K}$. Тогда

$$
\begin{gathered}
\bar{\alpha}_{i}^{1}\left(X^{K}, d\right)+\bar{\alpha}_{i}^{2}\left(X^{K}, d\right)=a_{i}(d), \quad \bar{\beta}_{i}^{1}\left(X^{K}, d\right)+\bar{\beta}_{i}^{2}\left(X^{K}, d\right)+1=b_{i}(d) \\
(d=1, \ldots, K, \quad i=3, \ldots, N(d)+1)
\end{gathered}
$$

ДокАЗАТЕЛЬСТво. Это утверждение следует из элементарных свойств раздутий и определения последовательностей $\bar{\alpha}_{i}^{1}\left(X^{K}, d\right)$ и $\bar{\beta}_{i}^{1}\left(X^{K}, d\right)$ для $d=1, \ldots, K$ и $i=3, \ldots$, $N(d)+1$. 
ЛЕмма 9. Пусть в обозначениях данного пункта

$$
\sum_{d=1}^{K} \frac{b_{N(d)+1}(d)}{a_{N(d)+1}(d)}<2-2 g(C)+e .
$$

Тогда

1) существуют положительные рачиональные числа $\lambda_{j}(d)$ и $\gamma$ такие, что выполнено соотношение

$$
K_{X^{K}} \equiv-2 C_{K}-\sum_{d=1}^{K} \sum_{j=1}^{N(d)+1} \lambda_{j}(d) \bar{F}_{j}(d)-\gamma F
$$

əде $\bar{F}_{j}(d)$ - неприводимая приведенная компонента слоя морфизма $\pi^{K}$ над точкой $x_{d}$, a $F-$ общий слой морфизма $\pi^{K}$;

2) $\partial$ ля $i=3, \ldots, N(d)$

$$
\frac{b_{i}(d)}{a_{i}(d)} \leqslant \frac{b_{N(d)+1}(d)}{a_{N(d)+1}(d)}
$$

3) форма пересечения кривых $C_{K}, \bar{F}_{r}(k)(k=1, \ldots, K$ u $r=1, \ldots, N(k))$ отрицательно определена.

ДокАЗАТЕЛЬСТво. 1) Пусть

$$
2-2 g(C)+e=\sum_{d=0}^{K} \varepsilon_{d}
$$

где $\varepsilon_{d} \in \mathbb{Q}_{>0}$ и $\varepsilon_{d}>b_{N(d)+1}(d) / a_{N(d)+1}(d)$ для $d=1, \ldots, K$. Тогда вьполнено соотношение $(4)$, в котором $\bar{F}_{j}(d)$ - неприводимая компонента слоя морфизма $\pi^{K}$ над точкой $x_{d}$, $F$ - общий слой морфизма $\pi^{K}$,

$$
\gamma=\varepsilon_{0}>0, \quad \lambda_{N(d)+1}(d)=a_{N(d)+1}(d) \varepsilon_{d}-b_{N(d)+1}(d)>0 \quad(d=1, \ldots, K) .
$$

Докажем, что $\lambda_{j}(d)>0$ для $d=1, \ldots, K$ и $j=1, \ldots, N(d)$. Если это не так, то для некоторого $k \in\{1, \ldots, K\}$ существует подмножество $\mathscr{J} \subset\{1, \ldots, N(k)\}$ такое, что $\bigcup_{j \subset \mathscr{J}} \bar{F}_{j}(k)$ связно и $\lambda_{j}(k) \leqslant 0$ для всех $j \subset \mathscr{J}$. Среди $\bar{F}_{j}(k)$ нет $(-1)$-кривых $(j \subset \mathscr{J})$, и форма пересечения кривых $\bar{F}_{j}(k)$ отрицательно определена (см. [4]). По формуле присоединения

$$
K_{X_{K}} \bar{F}_{j}(k)+\bar{F}_{j}(k)^{2} \geqslant-2 \quad(j \subset \mathscr{J}) .
$$

Следовательно, для всех $j \subset \mathscr{J} K_{X^{K}} \bar{F}_{j}(k) \geqslant 0$ и

$$
\begin{aligned}
0 \geqslant K_{X^{K}}\left(\sum_{j \subset \mathscr{J}} \lambda_{j}(k) \bar{F}_{j}(k)\right)= & \left.-2 C_{K}-\sum_{j=1}^{N(k)+1} \lambda_{j}(k) \bar{F}_{j}(k)\right)\left(\sum_{j \subset \mathscr{J}} \lambda_{j}(k) \bar{F}_{j}(k)\right) \\
= & -\left(\sum_{j \subset \mathscr{J}} \lambda_{j}(k) \bar{F}_{j}(k)\right)^{2}-2 C_{K}\left(\sum_{j \subset \mathscr{J}} \lambda_{j}(k) \bar{F}_{j}(k)\right) \\
& -\left(\sum_{j=1, j \not \subset \mathscr{J}}^{N(k)+1} \lambda_{j}(k) \bar{F}_{j}(k)\right)\left(\sum_{j \subset \mathscr{J}} \lambda_{j}(k) \bar{F}_{j}(k)\right) .
\end{aligned}
$$


Ho

$$
-\left(\sum_{j \subset \mathscr{J}} \lambda_{j}(k) \bar{F}_{j}(k)\right)^{2} \geqslant 0
$$

причем равенство выполнено, если $\lambda_{j}(k)=0$ для всех $j \subset \mathscr{J}$. Очевидно, что

$$
-2 C_{K}\left(\sum_{j \subset \mathscr{J}} \lambda_{j}(k) \bar{F}_{j}(k)\right) \geqslant 0
$$

и

$$
-\left(\sum_{j=1, j \not \subset \mathscr{J}}^{N(k)+1} \lambda_{j}(k) \bar{F}_{j}(k)\right)\left(\sum_{j \subset \mathscr{J}} \lambda_{j}(k) \bar{F}_{j}(k)\right) \geqslant 0 .
$$

Следовательно, для всех $j \subset \mathscr{J} \quad \lambda_{j}(k)=0$ и

$$
0 \leqslant\left(\sum_{j \subset \mathscr{J}} \bar{F}_{j}(k)\right) K_{X^{K}}=\left(\sum_{j \subset \mathscr{J}} \bar{F}_{j}(k)\right)\left(-2 C_{K}-\sum_{j=1}^{N(k)+1} \lambda_{j}(k) \bar{F}_{j}(k)\right)<0 .
$$

Значит, $\lambda_{j}(d)>0$ для $d=1, \ldots, K$ и $j=1, \ldots, N(d)$.

2) В части 1) было доказано, что если $2-2 g(C)+e=\sum_{d=0}^{K} \varepsilon_{d}$, где $\varepsilon_{d} \in \mathbb{Q}>0$ и $\varepsilon_{d}>b_{N(d)+1}(d) / a_{N(d)+1}(d)$ для $d=1, \ldots, K$, то выполнено соотношение $(4)$, в котором $\bar{F}_{j}(d)$ - неприводимая компонента слоя морфизма $\pi^{K}$ над точкой $x_{d}, F$ - общий слой морфизма $\pi^{K}$ и $\gamma=\varepsilon_{0}, \lambda_{j}(d)=a_{j}(d) \varepsilon_{d}-b_{j}(d)>0$ для $d=1, \ldots, K$ и $j=1, \ldots, N(d)$. При $\varepsilon_{d} \rightarrow b_{N(d)+1}(d) / a_{N(d)+1}(d)$ получаем нужное выражение.

3) Из 1), 2) следует существование положительных рациональных чисел $\lambda_{j}(d)$ и $\gamma$ таких, что вьполнено соотношение (4). Среди $C_{K}, \bar{F}_{r}(k)(k=1, \ldots, K, r=1, \ldots, N(k))$ нет (-1)-кривых, форма пересечения кривых $\bar{F}_{r}(k)(k=1, \ldots, K, r=1, \ldots, N(k))$ отрицательно определена (см. [4]) и

$$
C_{K}^{2} \leqslant C_{0}^{2}=-e<2-2 g(C)<0 .
$$

По формуле присоединения

$$
K_{X_{K}} C_{K}+C_{K}^{2} \geqslant 0, \quad K_{X_{K}} \bar{F}_{r}(k)+\bar{F}_{r}(k)^{2} \geqslant-2 .
$$

Следовательно,

$$
K_{X^{K}} \bar{F}_{r}(k) \geqslant 0 \quad(k=1, \ldots, K, \quad r=1, \ldots, N(k)), \quad K_{X^{K}} C_{K}>0 .
$$

Значит, для $k=1, \ldots, K$ и $r=1, \ldots, N(k)$

$$
0 \leqslant \bar{F}_{r}(k)\left(-2 C_{K}-\sum_{d=1}^{K} \sum_{j=1}^{N(d)+1} \lambda_{j}(d) \bar{F}_{j}(d)-\gamma F\right)
$$

и

$$
0<C_{K}\left(-2 C_{K}-\sum_{d=1}^{K} \sum_{j=1}^{N(d)+1} \lambda_{j}(d) \bar{F}_{j}(d)-\gamma F\right)
$$


Отсюда для $k=1, \ldots, K$ и $r=1, \ldots, N(k)$

$$
0 \leqslant \bar{F}_{r}(k)\left(-2 C_{K}-\sum_{d=1}^{K} \sum_{j=1}^{N(d)} \lambda_{j}(d) \bar{F}_{j}(d)\right), \quad 0<C_{K}\left(-2 C_{K}-\sum_{d=1}^{K} \sum_{j=1}^{N(d)} \lambda_{j}(d) \bar{F}_{j}(d)\right)
$$

и если $\bar{F}_{r}(k) \cap \bar{F}_{N(k)+1}(k) \neq \varnothing$, то

$$
0<\bar{F}_{r}(k)\left(-2 C_{K}-\sum_{d=1}^{K} \sum_{j=1}^{N(d)} \lambda_{j}(d) \bar{F}_{j}(d)\right) .
$$

Откуда следует, что форма пересечения кривых $C_{K}, \bar{F}_{r}(k)$ для $k=1, \ldots, K$ и $r=1, \ldots$, $N(k)$ отрицательно определена (см. [6]).

Лемма 10. В обозначениях данного пункта

$$
\sum_{d=1}^{K} \frac{b_{N(d)+1}(d)}{a_{N(d)+1}(d)}<2-2 g(C)+e
$$

тогда и только тогда, когда существует морфизм $f: X_{K} \rightarrow X$ такой, что

1) $X$ - численная поверхность Дель Пеичо;

2) $\operatorname{rk}(\operatorname{Div}(X) \otimes \mathbb{Q} / \equiv)=1$

3) $f$ стягивает кривые $C_{K}, \bar{F}_{j}(d)(d=1, \ldots, K, j=1, \ldots, N(d))$.

ДокАЗАТЕЛЬСтво. Необходимость. Пусть выполнено (5), тогда по лемме 9 на поверхности $X_{K}$ форма пересечения кривых $C_{K}, \bar{F}_{r}(k)$ для $d=1, \ldots, K$ и $j=1, \ldots, N(d)$ отрицательно определена. Существует морфизм $f: X_{K} \rightarrow X$, стягивающий кривые $C_{K}, \bar{F}_{j}(d)$ для $d=1, \ldots, K$ и $j=1, \ldots, N(d)($ см. [1]), и

$$
\operatorname{rk}\left(\operatorname{Div}\left(X_{K}\right) \otimes \mathbb{Q} / \equiv\right)=2+\sum_{d=1}^{K} N(d)
$$

следовательно, $\operatorname{rk}(\operatorname{Div}(X) \otimes \mathbb{Q} / \equiv)=1$. Легко видеть, что на поверхности $X$ вьполнено соотношение

$$
f_{*}\left(K_{X_{K}}\right)=K_{X} \equiv\left(\sum_{d=1}^{K} \frac{b_{N(d)+1}(d)}{a_{N(d)+1}(d)}-2-2 g(C)+e\right) f_{*}(F) .
$$

Из условия $\operatorname{rk}(\operatorname{Div}(X) \otimes \mathbb{Q} / \equiv)=1$ и

$$
\sum_{d=1}^{K} \frac{b_{N(d)+1}(d)}{a_{N(d)+1}(d)}-2-2 g(C)+e<0
$$

следует, что $X$ - численная поверхность Дель Пецщо.

Достаточность. Пусть существует морфизм $f: X_{K} \rightarrow X$ такой, что $X$ - численная поверхность Дель Пеццо, $\operatorname{rk}(\operatorname{Div}(X) \otimes \mathbb{Q} / \equiv)=1, f$ стягивает кривые $C_{K}, \bar{F}_{j}(d)$ 
$(d=1, \ldots, K, j=1, \ldots, N(d))$. Легко видеть, что на поверхности $X$ выполнено соотношение

$$
f_{*}\left(K_{X_{K}}\right)=K_{X} \equiv\left(\sum_{d=1}^{K} \frac{b_{N(d)+1}(d)}{a_{N(d)+1}(d)}-2-2 g(C)+e\right) f_{*}(F) .
$$

Из условия $\operatorname{rk}(\operatorname{Div}(X) \otimes \mathbb{Q} / \equiv)=1$ и того, что $X$ - численная поверхность Дель Пещц, следует

$$
\sum_{d=1}^{K} \frac{b_{N(d)+1}(d)}{a_{N(d)+1}(d)}-2-2 g(C)+e<0
$$

Tеорема 6. Существует взаимно однозначное соответствие мехду численными поверхностями Дель Пецио $X$ с нерациональными особенностями $и$ $\operatorname{rk}(\operatorname{Div}(X) \otimes \mathbb{Q} / \equiv)=1$ и следующей совокупностью:

1) линейчатая поверхность $\pi: \mathbb{P}_{C}\left(\mathscr{O}_{C} \oplus \mathscr{L}\right) \rightarrow C$ с инвариантом е, әде $\mathscr{L} \in$ $\operatorname{Pic}(C), g(C) \geqslant 1 u e=-\operatorname{deg}(\mathscr{L})>2 g(C)-2$

2) набор попарно различных точек $\left\{x_{1}, \ldots, x_{K}\right\} \subset C$, быть мохет, пустой $(K=0)$;

3) К последовательностей пар челых чисел

$$
\left(\alpha_{i}^{1}(d), \alpha_{i}^{2}(d)\right) \quad\left(d=1, \ldots, K, \quad i=3, \ldots, R(d)+1, \quad R(d) \in \mathbb{N}_{\geqslant 2}\right),
$$

которые удовлетворяют условию (*) и

$$
\sum_{d=1}^{K} \frac{\beta_{R(d)+1}^{1}(d)+\beta_{R(d)+1}^{2}(d)+1}{\alpha_{R(d)+1}^{1}(d)+\alpha_{R(d)+1}^{2}(d)}<2-2 g(C)+e,
$$

әде $\left(\beta_{i}^{1}(d), \beta_{i}^{2}(d)\right)$ - последовательность пар чельх чисел, двойственная $к$ $\left(\alpha_{i}^{1}(d), \alpha_{i}^{2}(d)\right)$ для $d=1, \ldots, K u i=3, \ldots, R(d)+1$.

Доказательство следует из теоремы 5 и лемм 7-10.

ЗАмечаниЕ 4. Теорема 6 дает не только классификацию всех численных поверхностей Дель Пещо с нерациональными особенностями и $\operatorname{rk}(\operatorname{Div}(X) \otimes \mathbb{Q} / \equiv)=1$, но и эффективньй алгоритм их построения. А именно, нужно

1) взять гладкую относительно минимальную линейчатую поверхность $\mathbb{P}_{C}\left(\mathscr{O}_{C} \oplus \mathscr{L}\right)$ с инвариантом $e$ и сечением $C_{0}$ такую, что $e-2 g(C)+2>0, \mathscr{L} \in \operatorname{Pic}(C)$, $C_{0}^{2}=-e=\operatorname{deg}(\mathscr{L})>2 g(C)-2$;

2) выбрать возможно пустой набор попарно различных точек $\left\{x_{1}, \ldots, x_{K}\right\} \subset C$;

3) сделать элементарное преобразование $\varphi: X_{K} \rightarrow \mathbb{P}_{C}\left(\mathscr{O}_{C} \oplus \mathscr{L}\right)$ в слоях морфизма $\pi$ над точками $\left\{x_{1}, \ldots, x_{K}\right\} \subset C$ так, чтобы $K$ последовательностей пар целых чисел

$$
\left(\alpha_{i}^{1}\left(X_{K}, d\right), \alpha_{i}^{2}\left(X_{K}, d\right)\right) \quad(d=1, \ldots, K, \quad i=3, \ldots, R(d)+1)
$$

удовлетворяли неравенству

$$
\sum_{d=1}^{K} \frac{\beta_{R(d)+1}^{1}(d)\left(X_{K}\right)+\beta_{R(d)+1}^{2}(d)\left(X_{K}\right)+1}{\alpha_{R(d)+1}^{1}(d)\left(X_{K}\right)+\alpha_{R(d)+1}^{2}(d)\left(X_{K}\right)}<2-2 g(C)+e
$$


где $\left(\beta_{i}^{1}(d), \beta_{i}^{2}(d)\right)(i=3, \ldots, R(d)+1)$ - последовательность пар целых чисел, двойственная к $\left(\alpha_{i}^{1}(d), \alpha_{i}^{2}(d)\right)$ для $d=1, \ldots, K$ и $i=3, \ldots, R(d)+1$, и $R(d)+1-$ число неприводимых компонент в слое морфизма $\pi \circ \varphi$ над точкой $x_{d} \in C$;

4) стянуть собственньй прообраз $C_{0}$ и все неприводимые компоненты слоев морфизма $\pi \circ \varphi$ над точками $\left\{x_{1}, \ldots, x_{K}\right\} \subset C$ кроме $(-1)$-кривых.

\section{СПИСОК ЦИТИРОВАННОЙ ЛИТЕРАТУРЫ}

[1] Sakai F. Weil divisors on normal surfaces // Duke Math. J. 1984. V. 51. P. 877-887.

[2] Fujisawa T. On non-rational Del Pezzo surfaces. Preprint TITECH-MATH 13-93 (№ 22). Tokyo: Tokyo Inst. Tech., 1993.

[3] Hartshorne R. Algebraic Geometry. Berlin: Springer Verlag, 1977.

[4] Beauville A. Surfaces Algebriques Complexes. V. 54. Montrouge: Astérisque, 1978.

[5] Brenton L. Some algebraicity criteria for singular surfaces // Invent. Math. 1977. V. 41. P. 129-147.

[6] Artin M. On isolated rational singularities of surfaces // Amer. J. Math. 1966. V. 88. P. 129-136. 